\title{
The Cardioprotective Effect of Combined Therapy With a-Lipoic Acid Preconditioning and Ischemic Postconditioning Is Mediated by the Improvement of Autophagy Flux and Mitochondrial Function in Myocardial Reperfusion Injury of Diabetic Rats
}

\section{Mahdi Abdoli Shadbad}

Tabriz University of Medical Sciences Faculty of Medicine

\section{Behnaz Mokhtari}

Tabriz University of Medical Sciences Faculty of Medicine

Alireza Alihemmati

Tabriz University of Medical Sciences Faculty of Medicine

Nasrin Abolhasanpour

Tabriz University of Medical Sciences

Aniseh Javadi

Tabriz University of Medical Sciences Faculty of Medicine

Reza Badalzadeh ( $\sim$ badalzadehr@tbzmed.ac.ir)

Tabriz University of Medical Sciences https://orcid.org/0000-0002-8092-7820

\section{Research Article}

Keywords: Autophagy, mitochondrial function, a-lipoic acid, ischemic postconditioning, diabetic heart, ischemia/reperfusion injury

Posted Date: July 29th, 2021

DOI: https://doi.org/10.21203/rs.3.rs-719347/v1

License: (c) (i) This work is licensed under a Creative Commons Attribution 4.0 International License.

Read Full License 


\section{Abstract}

Investigating the interaction of diabetes with ischemic postconditioning (IPostC)-associated cardioprotection in myocardial ischemia/reperfusion (I/R) damage is of great clinical importance. The present work was performed to evaluate the combined effects of a-lipoic acid (LA) and IPostC on autophagy flux and mitochondrial function following myocardial I/R damage in type-ll diabetic rats. Diabetes with duration of 12 weeks was induced by high-fat diet/low dose of streptozotocin. LA (500 $\mathrm{mg} / \mathrm{kg} / \mathrm{day}$ ) was administered orally in diabetic rats for 5 weeks before l/R. The hearts were removed and mounted on Langendorff apparatus. I/R was induced through the ligation of left anterior descending coronary artery for $35 \mathrm{~min}$ and reperfusion for $60 \mathrm{~min}$. IPostC was applied immediately at the onset of the reperfusion. Lastly, myocardial infarct size (IS), autophagy markers at both gene and protein levels, and mitochondrial ROS production and membrane potential were assessed. Combination of LA and IPostC significantly decreased the IS of diabetic hearts $(P<0.05)$. IPostC alone could not significantly decrease p62 gene and protein expressions, and mitochondrial membrane depolarization in diabetic hearts. However, combination of LA and IPostC more significantly decreased LC3 and p62 gene expressions ( $\mathrm{P}<$ $0.01)$, LC3II/LC3I and p62 protein expressions $(P<0.01)$, and mitochondrial ROS generation and membrane depolarization $(P<0.01)$ in diabetic hearts. Pretreatment with LA in diabetic rats notably restored cardioprotection by IPostC via modulation of autophagy flux and restoring mitochondrial function. This combined conditioning might be an effective strategy to diminish I/R injury in diabetic hearts.

\section{Introduction}

Ischemic heart disease (IHD) is the leading cause of death in diabetic patients [1]. Timely restoration of blood flow to the ischemic area, which means reperfusion, is an effective therapeutic strategy in which decreases the mortality rate, but also leads to ischemia/reperfusion (I/R) injury [2]. Recently, we and others showed that diabetes increases the susceptibility of the heart to I/R damage, exacerbates cardiac injury, and results in poor prognosis in patients with myocardial infarction (MI) through multiple mechanisms that are largely unknown. In other words, the risk of mrocardial I/R development in diabetic patients is higher than non-diabetic ones [3-5]. It has been revealed that hyperglycemia, increased production of fatty acids, impaired production of nitric oxide, overproduction of free radicals and lipid peroxidation, impaired activation of survival protein kinases, mitochondrial dysfunction, and autophagic dysregulation may be involved in the interaction of diabetes with cardioprotective mechanisms under I/R damage $[3,4,6]$.

Autophagy as an intracellular catabolic pathway has house-keeping role in maintaining cellular homeostasis via the degradation of long-lived and misfolded proteins, as well as dysfunctional and injured organelles such as mitochondria. The dual roles of autophagy in the regulation of cell death and survival have been shown in preclinical studies of diabetic myocardium with I/R damage, but the protective versus detrimental effects of altered autophagy in myocardial I/R injury, especially in the presence of cardiovascular risk factors such as diabetes still requires further investigations $[7,8]$. It has 
been suggested that by inducing well-controlled autophagy at a proper level and timing, and inhibiting unsuitable autophagy, the heart can be protected from I/R injury [5]. Given that there is a potential association between altered autophagy and mitochondrial dysfunction, autophagy plays an essential role in maintaining the normal structure and function of mitochondria in the heart [9]. Mitochondrial dysfunction is involved in the poor prognosis of IHD in diabetic condition, because diabetes impairs mitochondrial biogenesis in the heart, and decreases the function and number of mitochondria, which are ultimately associated with cardiac dysfunction [5]. So improving mitochondrial biogenesis and reducing mitochondrial oxidative stress have emerged as potential targets for reducing I/R injury in diabetic hearts $[10,11]$.

Ischemic postconditioning (IPostC) is considered as a protective endogenous mechanism against myocardial ischemia. Because the diabetic heart is more resistant to common cardioprotections, therapeutic strategies such as IPostC which have shown positive effects in reducing myocardial I/R injury in non-diabetic experimental models have failed to reduce I/R injury in diabetic hearts. Because diabetes eliminates the positive effects of IPostC against myocardial I/R damage via interfering with the cell survival signaling pathways $[6,12]$. Emerging data suggests that optimal cardioprotection in the diabetic hearts subjected to I/R may be achieved by combination therapy. Therefore, enhancing the effectiveness of IPostC in order to reduce reperfusion injury under diabetic conditions, and restoring the loss of cardioprotection induced by diabetes, its combination therapy with appropriate pharmacological agents has been proposed in several studies $[3,4]$.

a-lipoic acid (LA) is a potent natural antioxidant and free radical scavenger which has beneficial effects in preventing cardiovascular diseases [13]. It has been shown that LA has the ability to increase cellular defense and elevate the resistance to ROS-induced myocardial damage [14]. In addition, LA has a wide range of pharmacological capabilities in diabetic patients including glucose and lipid-lowering effect, insulin-sensitizing, and anti-oxidative, anti-apoptotic and anti-inflammatory effects [15]. Given the therapeutic potentials of LA in cardiovascular diseases as well as in diabetes, we evaluated the effects of combination therapy with LA preconditioning and IPostC in type-II diabetic hearts injured by I/R by highlighting the role of autophagy and mitochondrial function in this context in order to achieve more effective and better cardioprotection.

\section{Materials And Methods \\ 2.1. Chemicals}

Streptozotocin (STZ) was supplied from Sigma (St.Louis, MO, USA), LA was obtained from Novartis (Switzerland), all materials of Krebs-Henseleit $(\mathrm{K}-\mathrm{H})$ solution were purchased from Merck Company (Munchen, Germany), and all other chemicals and reagents were supplied from commercial sources in the highest quality available.

\subsection{Animals}


Male Wistar rats (84 rats at 8 weeks of age, $200-250 \mathrm{~g}$ ) were obtained from the animal center of Tabriz University of Medical Sciences. The rats were kept in the individual cages in the standard animal room with controlled temperature $\left(20-24^{\circ} \mathrm{C}\right)$ and humidity (55\%) under $12 \mathrm{hr}$ dark-light cycles, and had free access to food and water ad libitum. All procedures of this research project were carried out in accordance with the Guide for the Care and Use of Laboratory Animals published by the US National Institutes of Health (8th Edition, NRC 2011) and were approved by the ethical committee of Tabriz University of Medical Sciences (Ethical code: IR.NIMAD.REC.1396.029).

\subsection{Induction of type-II diabetes}

In order to induce type-II diabetes with insulin resistance, the method of high-fat diet-fed and low-dose STZ was used in accordance with the previous studies [16]. For simulation of chronic diabetes, the diabetic period was continued for 12 weeks. After the adaptation of animals for one week, diabetic rats received ad libitum with a high-fat diet (0.3\% DL-Methionine, $1 \%$ cholesterol, $4 \%$ sucrose, $24 \%$ casein, $30 \%$ lard and $35 \%$ normal pellet) for 6 weeks (62\% calories was from fat, and total caloric value was about 4.6 $\mathrm{kcal} / \mathrm{g})$. At the end of the 6 th week, STZ in low dose $(35 \mathrm{mg} / \mathrm{kg}$ in citrate buffer, $\mathrm{pH} 4)$ was injected intraperitoneally (i.p) in diabetic rats following $8 \mathrm{hr}$ fasting. For confirmation of diabetes, blood samples were collected $72 \mathrm{hr}$ following the injection of STZ, and the levels of blood glucose were determined by means of a glucometer device (elegance CT-X12, Convergent Technologies, Germany). The animals with blood glucose levels higher than $250 \mathrm{mg} / \mathrm{dl}$ were considered as diabetic. Non-diabetic healthy rats were fed with typical food and received an equal volume of citrate buffer. Following 6 weeks of diabetes induction, the diabetic as well as non-diabetic rats were sacrificed and all the experiments were done in the isolated perfused hearts.

\subsection{Isolated heart perfusion and induction of regional I/R}

All rats were heparinized (500 IU/kg) to avoid blood clotting during surgery, and anesthetized by i.p injection of a mixture of ketamine $(60 \mathrm{mg} / \mathrm{kg})$ and xylazine $(10 \mathrm{mg} / \mathrm{kg})$. Then the hearts were rapidly excised, and immersed in icecold $\mathrm{KH}$ solution, and connected to the perfusion cannula via the aorta and mounted on a pressure-constant Langendorff perfusion apparatus (ML176-V; AD Instruments, New South Wales, Australia), in which the isolated hearts were retrogradely perfused via the aorta with $\mathrm{K}-\mathrm{H}$ solution containing (in mmol/L) KCl 4.7; $\mathrm{NaCl} 118 ; \mathrm{KH}_{2} \mathrm{PO}_{4} 1.2 ; \mathrm{MgSO}_{4}$ 1.2; $\mathrm{CaCl}_{2} 2.5 ; \mathrm{NaHCO}_{3} 25$; and glucose 11.1 at a constant perfusion pressure of $80 \mathrm{~mm} \mathrm{Hg}$ and pH 7.4. The perfusion solution was gassed with a mixture of $95 \% \mathrm{O}_{2}$ and $5 \% \mathrm{CO}_{2}$ at $37^{\circ} \mathrm{C} \pm 0.5^{\circ} \mathrm{C}$. The isolated heart was surrounded by a homeothermic glass cover in order to preserve the temperature near $37^{\circ} \mathrm{C}$. As stabilization period, the isolated hearts of all groups were perfused with K-H solution for $15 \mathrm{~min}$, and the coronary flow rate was $12-14 \mathrm{ml} / \mathrm{min}$. Then, the isolated hearts were subjected to regional ischemia for $35 \mathrm{~min}$ by occluding left anterior descending ( $L A D$ ) coronary artery using a $5-0$ silk ligature placed around the $L A D$ close to its origin, followed by reperfusion for 60 min by re-opening of LAD. The Sham-operated groups underwent the above procedure, without LAD ligation. Coronary flow was assessed by timed collection of coronary effluent. The efficiency of coronary ligation and sufficient perfusion were approved by an immediate fall in coronary flow at the onset of index ischemia to about $30-40 \%$ of its baseline value and the recovery of 
the coronary flow upon reperfusion. IPostC in corresponding groups was induced by 6 cycles of $10 \mathrm{sec}$ ischemia/10 sec reperfusion, applied immediately at the onset of reperfusion.

\subsection{Study design}

The rats were randomly divided into 2 non-diabetic and 5 diabetic subgroups ( $n=12 /$ each). The nondiabetic healthy $(\mathrm{H})$ rats were divided into $\mathrm{H}$-Sham (non-diabetic hearts receiving 110 min full perfusion), and $\mathrm{H}$-Cont (non-diabetic hearts receiving $35 \mathrm{~min}$ ischemia plus $60 \mathrm{~min}$ reperfusion). The diabetic (D) rats included D-Sham (diabetic hearts receiving $110 \mathrm{~min}$ full perfusion), D-Cont (diabetic hearts receiving 35 min ischemia plus 60 min reperfusion), D-Post (diabetic hearts receiving 35 min ischemia plus 6 cycles of $10 \mathrm{sec}$ ischemia/10 sec reperfusion at the beginning of reperfusion), D-LA (the hearts of LA-receiving diabetic rats undergoing $35 \mathrm{~min}$ ischemia plus $60 \mathrm{~min}$ reperfusion), and D-Post-LA (the hearts of LAreceiving diabetic rats undergoing $35 \mathrm{~min}$ ischemia plus 6 cycles of $10 \mathrm{sec}$ ischemia/10 sec reperfusion at the beginning of reperfusion).

LA (500 mg/ $\mathrm{kg} /$ day) was administered in D-LA and D-LA-Post groups through feeding gavage in the last 5 weeks. Body weight recording was performed weekly, with the final body weight recording prior to excision and isolation of the heart. The ratio of heart/body weight (\%) was measured as an index of diabetic cardiomyopathy.

\subsection{Assessment of HOMA1-IR index}

Fasting blood glucose and fasting plasma insulin levels were evaluated for confirmation of insulin resistance and type-Il diabetes. Before isolation of the hearts, blood samples were collected from the hearts and immediately centrifuged for separation of plasma $\left(3500 \times \mathrm{g}, 10 \mathrm{~min}, 4^{\circ} \mathrm{C}\right)$. Then, plasma levels of insulin were measured using a rat-specific enzyme-linked immunosorbent assay (ELISA) kit (Cayman Chem., Ann Arbor, Michigan) according to the manufacturer's instructions. In addition, the levels of fasting blood glucose were determined using a glucometer device. Homeostasis model assessment of insulin resistance (HOMA1-IR) index was used in order to identify insulin resistance using the following formula: fasting blood glucose $(\mathrm{mmol} / \mathrm{l}) \times$ fasting insulin $(\mu \mathrm{u} / \mathrm{l}) / 22.5[17]$.

\subsection{Assessment of infarct size}

At the end of 60 min reperfusion, the LAD was occluded again and $2 \mathrm{ml}$ Evans blue dye $(0.25 \%)$ was infused through the aortic root into the coronary system. Next, the hearts were excised and frozen at $-20^{\circ} \mathrm{C}$. After $24 \mathrm{~h}$, thin slices $(2 \mathrm{~mm})$ from the apex to the base were prepared and incubated in 2,3,5triphenylte-trazolium chloride (TTC, $1 \%$ ) in phosphate-buffered solution ( $\mathrm{pH} \mathrm{7.4)}$ for $15-20 \mathrm{~min}$ at $37^{\circ} \mathrm{C}$. Then, the slices were placed in 10\% formalin for 24-48 $\mathrm{h}$ to improve the contrast of the staining. For calculation of area at risk and infarct size (IS) of each slice, Image $\mathrm{J}$ software ( $\mathrm{NIH}$, Bethesda, USA) was used. The area at risk was expressed as a percentage of left ventricle (AAR/LV $\times 100)$. The infarct size was expressed as a percentage of area at risk (IS/AAR $\times 100)$.

\subsection{Quantitative real-time PCR}


Total RNA was extracted from $100 \mathrm{mg}$ of fresh samples of LVs which were immersed in RNase Later solution, using the Trizol Reagent (Invitrogen Company, San Diego, CA, USA) on ice, in accordance to the manufacturer's instructions. In order to detect the RNA yield and purity, NanoDrop spectrophotometer at wavelength of $260 / 280 \mathrm{~nm}$ (NanoDrop ND-2000C, Thermo Fisher Scientific, USA) was employed. Using an Exiqon cDNA Synthesis Kit, first-strand complementary DNA (CDNA) was synthesized from the obtained samples' RNAs. By means of a LightCycler-96 Roche device, the assessment of the gene expressions of microtubule-associated proteins 1A/1B light chain 3 (LC3) and p62 were performed. The production of forward and reverse primers of genes was made by the custom oligonucleotide synthesis service Metabion (Martinsried, Germany). Primers designing were performed by Primer-3 software. In order to check out the primers specificity, primers were blasted via Basic Local Alignment Search Tool on the NCBI website (http://www.ncbi.nlm.nih.gov/tools/primer-blast/). The specific primers sequences of the present work are listed in Table 1 . The confirmation of purity of amplified products was performed by using melting curve and analyzed to ensure the identity of the specific PCR product. Glyceraldehyde 3phosphate dehydrogenase (GAPDH) was the internal control for this study. $2^{-\Delta \Delta C T}$ method was used for determining the relative-quantitative levels of autophagy genes. The results were expressed as foldchange difference to the relevant controls.

Table 1

Primer sequences for quantitative real-time PCR analysis.

\begin{tabular}{|lll|}
\hline Gene name & Primer sequence & PCR product size (bp) \\
\hline LC3 & $\begin{array}{l}\text { Forward: 5'-GGGTGGATTAGGCAGAGATGTGA-3' } \\
\text { Reverse: 5'-GATGAGGGGCAAGATGGGTAGA-3' }\end{array}$ & \\
\hline p62 & $\begin{array}{l}\text { Forward: 5'-TCCAGCACAGGCACAGAAGAC-3' } \\
\text { Reverse: 5'-CCGACTCCATCTGTTCCTCTG-3' }\end{array}$ & \\
& Forward: 5'-AAGTTCAACGGCACAGTCAAGG-3' & 121 \\
GAPDH & Reverse: 5'-CATACTCAGCACCAGCATCACC-3' & \\
\hline
\end{tabular}

\subsection{Western blot assay}

At 60 min after reperfusion, the expression of LC3II/LC3I and p62 were determined in the LVs using western blot assay. After dissecting and homogenizing fresh-frozen samples in radioimmunoprecipitation assay (RIPA) lysis buffer (Sigma-Aldrich, St. Louis, MO, USA), the resulting solutions were centrifuged at $10,000 \mathrm{RCF}$ for $10 \mathrm{~min}$ at $4^{\circ} \mathrm{C}$. Next, the supernatants were collected. The determination of total protein concentration was performed using UV 3000 ultraviolet spectrophotometer (NanoDrop, Wilmington, DE). After the loading of equal amounts of proteins $(50 \mu \mathrm{g})$ into the electrophoresis chamber in 10-15\% SDS-PAGE, separated soluble proteins were transferred to a polyvinylidene difluoride (PVDF, Sigma-Aldrich, St. Louis, MO, USA) membrane. Incubation of membranes with $5 \%$ non-fat dry milk solution in Tris buffered saline-Tween 20 (TBST, pH 7.4) for $2 \mathrm{~h}$ at room temperature were employed for blocking non-specific bindings. The membranes were then incubated with 
primary antibodies at 1:1000 against LC3B (1:1000, Cell Signaling) and p62/SQSTM1 (1:500, Santa Cruz) diluted in blocking buffer overnight at $4^{\circ} \mathrm{C}$ on a shaker. The membranes were also incubated for $\beta$-actin antibody (1:500, Cell Signaling), diluted in TBST overnight at $4^{\circ} \mathrm{C}$ in order to control for equal loading. Next, membranes were incubated with horseradish peroxidase-conjugated secondary antibody (1:7000, Cell Signaling) for $1 \mathrm{~h}$ on a shaker at room temperature. The membranes were rinsed with wash buffer at least $3 \times 10 \mathrm{~min}$, and were incubated with enhanced chemiluminescence (ECL) reagents (Amersham) in order to detect the immunoreactivity, and then were exposed to X-ray hyperfilm inside a hypercassette in a darkroom for $5 \mathrm{~min}$. The chemiluminescence of the antibody bindings was visualized using a visualizing machine. Protein bands intensity in the blots was quantified by densitometric analysis and normalized with the intensity of $\beta$-actin bands as internal control. The measured values are presented in arbitrary unit.

\subsection{Evaluation of mitochondrial function}

Isolation of cardiac mitochondri was performed according to our prevous studies $[18,19]$. The production of cardiac mitochondrial ROS was evaluated by dichlorohydro-fluorescein diacetate (DCFDA) dye using a fluorometric method. The changes of mitochondrial membrane potential in the heart were evaluated using the 5,5',6,6'-tetrachloro-1,1',3,3'-tetraethylbenzimidazolylcarbocyanine iodide (JC-1) by an isolated mitochondrial staining kit (Sigma, Germany), in accordance with the manufacturer's protocol [18].

\subsection{Statistical analysis}

All quantitative data were expressed as means \pm standard errors of the mean (SEM). Statistical analysis of data was carried out using the SPSS software 25 (SPSS Inc., Chicago, IL, USA). Differences between groups were analysed by one-way ANOVA followed by Tukey post hoc test. $\mathrm{P}<0.05$ was considered statistically significant.

\section{Results}

\subsection{Characteristics of the animals}

The statistical analyses showed significant increases in the levels of fasting blood glucose, plasma levels of insulin, HOMA1-IR index, heart weight (HW) and the ratio of heart/body weight (HW/BW) as compared to the non-diabetic rats $(P<0.01$ for all). Pretreatment of diabetic rats with LA significantly decreased hyperglycemia $(P<0.01)$, increased the plasma levels of insulin $(P<0.05)$, and reduced HOMA-IR index $(P$ $<0.05), \mathrm{HW}(\mathrm{P}<0.05)$, and HW/BW ratio $(P<0.05)$ as compared to the diabetic rats $($ Table 2$)$. 
Table 2

General information of experimental groups.

\begin{tabular}{|c|c|c|c|}
\hline Groups & Non-diabetic & Diabetic & Diabetic + LA \\
\hline Fasting blood glucose (mg/dl) & $96 \pm 4.4$ & $540 \pm 15^{\star \star}$ & $346 \pm 12^{\star \star,++}$ \\
\hline Insulin ( $\mu \mathrm{u} / \mathrm{ml})$ & $4.7 \pm 0.5$ & $7.2 \pm 0.6^{\star \star}$ & $9.3 \pm 0.4^{\star *,+}$ \\
\hline HOMA1-IR & $1.1 \pm 0.1$ & $9.3 \pm 0.2^{* *}$ & $8.2 \pm 0.5^{\star *,+}$ \\
\hline BW (g) & $295 \pm 11$ & $311 \pm 14$ & $305 \pm 9$ \\
\hline HW (g) & $1.36 \pm 0.03$ & $1.61 \pm 0.04^{\star \star}$ & $1.48 \pm 0.06^{+}$ \\
\hline HW/BW (\%) & $0.46 \pm 0.01$ & $0.53 \pm 0.01^{\star *}$ & $0.49 \pm 0.01^{+}$ \\
\hline
\end{tabular}

\subsection{Myocardial area at risk and infarct size}

As shown in Fig. 1. A, there were no significant differences in the area at risk between all of the hearts subjected to $35 \mathrm{~min}$ ischemia and 60 min reperfusion. As shown in Fig. 1. B, induction of $35 \mathrm{~min}$ ischemia and 60 min reperfusion in non-diabetic rats caused the development of infarct size to $39 \pm 3.2 \%$, and this value in the diabetic rats was $47 \pm 8.1 \%$. So there was no significant difference between $\mathrm{H}$-Cont and D-Cont groups. Postconditioning with ischemia alone or preconditioning with LA alone could not significantly reduced the IS in comparison to those of non-treated diabetic hearts. However, combined therapy with IPostC and LA significantly decreased the IS compared to D-Cont group $(P<0.05)$.

\subsection{The expression of LC3 and p62 genes}

As shown in Fig. 2. A, the expression of LC3 gene in H-Cont group was increased to some extent as compared with H-Sham group, however there was no significant difference between them. In diabetic hearts, the expression of LC3 gene was significantly increased in D-Sham group as compared with $\mathrm{H}$ Sham group $(P<0.01)$. In addition, the expression of LC3 gene in D-Cont group was significantly increased as compared with $\mathrm{H}$-Sham $(P<0.01)$ and $\mathrm{D}$-Sham $(\mathrm{P}<0.05)$ groups. Treatment of diabetic hearts decreased the expression of LC3 gene. In details, this reduction was statistically significant in DPost $(P<0.05), D-L A(P<0.01)$, and D-Post-LA $(P<0.01)$ in comparison with $D$-Cont group. Furthermore, LA preconditioning alone or in combination with IPostC significantly decreased the expression of LC3 gene as compared with $D$-Post group ( $P<0.05$ for both).

As shown in Fig. 2. B, the expression of p62 gene in $\mathrm{H}$-Cont group was significantly increased as compared with $\mathrm{H}$-Sham group $(\mathrm{P}<0.05)$. In diabetic hearts, the expression of p62 gene was significantly increased in $\mathrm{D}$-Sham group as compared with $\mathrm{H}$-Sham group $(\mathrm{P}<0.01)$. In addition, the expression of p62 
gene in D-Cont group was significantly increased as compared with H-Sham group $(P<0.01)$, as well as increased to some extent as compared with D-Sham group, however there was no significant difference between them. IPostC alone could not significantly decrease the expression of p62 gene in diabetic hearts as compared with D-Cont group. But LA preconditioning alone or in combination with IPostC significantly decreased the expression of p62 gene as compared with $D$-Cont group $(P<0.05$ and $P<0.01$, respectively).

\subsection{LC3II/LC3I and p62 protein levels}

As shown in Fig. 3. A, LC3II/LC3I in H-Cont group was increased to some extent in comparison with HSham group, but this difference was not significant between them. Also, LC3II/LC3I expression in D-Sham group was significantly higher than $\mathrm{H}$-sham group $(\mathrm{P}<0.01)$. In addition, LC3II/LC3I in D-Cont group was significantly increased as compared with $\mathrm{H}$-Sham $(P<0.01)$ and $D$-Sham $(P<0.05)$ groups. Treatment of diabetic hearts decreased LC3II/LC3I expression. In details, this reduction was statistically significant in

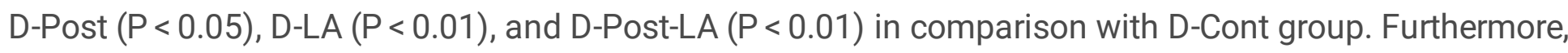
LA preconditioning alone or in combination with IPostC significantly decreased LC3II/LC3I as compared with $\mathrm{D}$-Post group $(\mathrm{P}<0.05$ for both).

As shown in Fig. 3. B, protein expression of p62 in H-Cont group was increased to some extent as compared with $\mathrm{H}$-Sham group, however there was no significant difference between them. Also, the expression of p62 protein in D-Sham group was significantly increased as compared with H-Sham group $(P<0.05)$. In addition, protein expression of $p 62$ in $D$-Cont group was significantly increased as compared with H-Sham $(P<0.01)$ and D-Sham $(P<0.05)$ groups. IPostC alone could not significantly decrease protein expression of p62 in diabetic hearts as compared with D-Cont group. But LA preconditioning alone or in combination with IPostC significantly decreased protein expression of p62 as compared with $D$-Cont group $(P<0.05$ and $P<0.01$, respectively). Furthermore, LA preconditioning in combination with IPostC significantly decreased protein expression of p62 as compared with D-Post group $(P<0.05)$.

\subsection{Mitochondrial function}

Figure 4. A shows the levels of mitochondrial ROS production in the heart, which were reported as fluorescence intensity of DCF. As shown, the levels of ROS production in $\mathrm{H}$-Cont hearts were significantly higher as compared to H-Sham hearts $(P<0.05)$. In addition, ROS production levels in $D$-Cont group were significantly higher as compared with H-Sham $(P<0.01)$ and D-Sham $(P<0.05)$ groups. Interestingly, the levels of mitochondrial ROS production were significantly decreased in D-Post $(P<0.01), D-L A(P<0.05)$, and D-Post-LA $(P<0.01)$ groups as compared with $D$-Cont group.

Figure 4. B shows the alterations of mitochondrial membrane potential in the heart, which were reported as red/green fluorescent intensity ratio of JC-1 staining. Lack of unwanted depolarization or normal membrane potentials associate with greater red/green ratios. The ratio of red/green fluorescent intensity was significantly lower in $\mathrm{H}$-Cont group as compared to $\mathrm{H}$-Sham group $(\mathrm{P}<0.01)$. Also, this ratio in $\mathrm{D}$ Sham group was significantly lower as compared with $\mathrm{H}$-Sham group $(P<0.05)$. In addition, this ratio in D-Cont group was significantly lower as compared with H-Sham $(P<0.001)$ and D-Sham $(P<0.01)$ 
groups. IPostC alone could not significantly increase the ratio of red/green fluorescent intensity in diabetic hearts as compared with D-Cont group. However, LA preconditioning alone or in combination with IPostC significantly increased this ratio as compared with $D$-Cont group $(P<0.01$ for both) and $D$ Post group ( $P<0.05$ and $P<0.01$, respectively).

\section{Discussion}

The present experimental study showed the effectiveness of combined therapy with LA preconditioning and IPostC in type-II diabetic hearts injured by I/R by investigating autophagy flux and mitochondrial function. Pretreatment of type-II diabetic rats with LA restored the cardioprotective effects of IPostC in the hearts with I/R injury, as evidenced by decreased myocardial infarct size. The reduction of myocardial infarct size by combined conditioning was partly mediated by its influence on autophagy flux and mitochondrial function following reperfusion injury in diabetic hearts. Our data documented that this combined therapy suppressed the excessive autophagy flux via reducing LC3II and p62 at both gene and protein levels, as well as decreased the generation of mitochondrial ROS and mitochondrial membrane depolarization.

Studies have shown that diabetes mellitus leads to the aggravation of heart function after I/R injury, and reduction of cardioprotective strategies efficiency [1]. The characteristics of type-II diabetes includes glucose intolerance, hyperglycemia, hyperinsulinemia, insulin resistance, and hyperlipidemia [7]. In this study, using high-fat diet/streptozotocin (HFD/STZ) in rats resulted in hyperglycemia, hyperinsulinemia, insulin resistance, and an increased HW/BW ratio. In details, at the end of 7th week, the diabetic rats showed glucose intolerance, and at the end of 12th week showed high fasting blood glucose, hyperinsulinemia and increased HOMA1-IR. Accordingly, these results confirmed that the rats underwent type-II diabetes and insulin resistance. Surprisingly, preconditioning with LA decreased blood glucose levels to the normal state, elevated insulin sensitivity, and reduced HW/BW ratio in diabetic animals. In agreement with our findings, a previous study showed that LA has positive effects against diabetic cardiomyopathy development by inhibition of mitochondrial oxidative injury through decreasing cardiomyocyte apoptosis and increasing the levels of GSH and the activity of SOD [14]. Several previous studies have suggested that diabetes can increase cardiac susceptibility to I/R damage, leading to the increased infarct size $[20,21]$. Inconsistent, this model of chronic diabetes in our study tends to increase the infarct size in I/R hearts compared with non-diabetic hearts, but this difference was not significant. The contrary of our finding can be described by the possible ability of preceding diabetes in increasing the cardiac resistance against I/R injury due to its possible preconditioning effects via producing several humoral mediators, but requires further evaluations $[12,22]$. It has been revealed that diabetic heart leads to the excessive generation of mitochondrial ROS, reduction of NO levels, endothelial dysfunction, myocardial inflammation, apoptosis, dysregulated autophagy, and consequently contractile dysfunction $[23,24]$.

Autophagy is critical in degradation of old damaged cellular organelles and proteins, and has an important role in physiological function of the heart through maintaining cellular homeostasis [25]. Under 
myocardial I/R injury, autophagy plays both destructive and beneficial roles, which depends on the level of autophagy activity [5]. Thus, maladaptive or adaptive functions of autophagy under I/R condition is still under discussion. It has been demonstrated that increase or decrease in autophagy activity can induce cell death via excessive degradation of vital organelles and proteins, or by accumulation of injured organelles and proteins, respectively [26, 27]. Several preclinical studies reported that induction of adaptive autophagy in the heart may be protective under I/R injury [28-30]. On the other hand, there are preclinical studies demonstrating that reduction of autophagy activity in the heart may be protective under I/R injury [31-33]. Under diabetic condition, metabolic abnormalities such as hyperglycemia, insulin resistance, dyslipidemia, and excessive ROS can inhibit or induce autophagy process in the myocardium, thus lead to the diabetic cardiomyopathy development [7]. It has been proven that impaired autophagy in the heart of animals with HFD-induced insulin resistance and type-ll diabetes could be occurred at the baseline, as well as following ischemia [34]. In the present work, I/R injury caused the increased LC3II and p62 in the heart at both gene and protein levels under diabetic condition. It should be noted that because of some limitations, the autophagy flux was not assessed in our study. However, according to the related data of this study it can be said that impaired autophagy flux following I/R in diabetic hearts occurred, that is in agreement with previous studies. In details, it seems that following I/R injury in non-diabetic hearts autophagy tends to be slightly unregulated. In addition, LC3 and p62 gene expressions, as well as LC3II/LC3I and p62 protein levels were significantly increased in diabetic hearts with I/R injury. So it can be indirectly demonstrated that type-II diabetes led to the unregulation of autophagy. In other words, according to the previous studies and our data, it was more likely that type-II diabetes led to the impairment of autophagy flux at the late stage of lysosomal degradation, as evidenced by increased levels of p62 in diabetic hearts with I/R.

Mitochondrial dysfunction has been associated with insulin resistance, and is considered as a major target of oxidative damage in diabetic heart injury. Excessive production of ROS from injured mitochondria which causes cardiomyocyte death through releasing pro-death factors, and subsequent autophagosome accumulation, are commonly observed in diabetic myocardium. Autophagy has a key role in reduction of diabetic heart injury through elimination of dysfunctional or damaged mitochondria, thereby prevention of ROS generation [35-37]. Similarly, our study showed that I/R injury caused increased levels of ROS production in the heart under normal and diabetic conditions. In addition, reduction of mitochondrial membrane potential which indicates more depolarization was observed in non-diabetic and diabetic hearts with I/R injury. These may reflect the fact that targeting novel strategies in order to prevent excessive generation of ROS by removing dysfunctional mitochondria via timly restoring the autophagy flux in appropriate level in the diabetic myocardium with I/R injury would be useful.

Accordingly, in the current work, application of LA alone caused significant reduction of LC3II and p62 at both gene and protein levels in one hand, and decreased mitochondrial ROS production and improved mitochondrial membrane potential on the other hand. Also, application of IPostC alone significantly reduced LC3 gene expression, LC3II/LC3I protein levels, and mitochondrial ROS generation. However, preconditioning with LA alone, or postconditioning with ischemia alone could not significantly decrease 
myocardial infarct size. It can be probably explained with this hypothesis that other protective mechanisms may not be influenced by single therapy with LA or IPostC in diabetic hearts with I/R injury. Nevertheless, additional investigations are needed to evaluate this hypothesis's reliance. The current work showed that combined therapy with LA and IPostC decreased the infarct size of diabetic heart. This positive effect of combined therapy on infarct size was in consistent with its effects on restoring autophagy flux and improving mitochondrial function. Of note, the effects of combined therapy with LA and IPostC on autophagy flux and mitochondrial function were more effective and potent as compared to the single therapy with them. In other words, addition of LA with IPostC elevated the efficacy of IPostC to restore autophagy flux and mitochondrial function in diabetic I/R hearts. Taken together, it seems that diabetes interferes with cardioprotective effects of IPostC, and possibly reduces its efficacy against myocardial I/R damage. It seems that application of LA raises the potency and efficacy of IPostC in diabetic hearts likely via normalization of some diabetes-associated cellular alterations. This finding suggests that combined conditioning may possibly lead to the effective co-activation of multiple mechanisms of cardioprotection, which requires further clarification. In agreement with the present results, our previous works also confirmed that IPostC alone was not able to decrease infarct size and improve cardiac function following I/R damage under diabetic condition. However, additioning an appropriate therapeutic agent with IPostC caused more cardioprotective effects [3, 19]. In addition, combined therapy with vildagliptin and IPostC had cardioprotective effects via restoration of autophagy flux and improving mitochondrial function in diabetic hearts injured with I/R [19].

In conclusion, combined therapy with LA and IPostC induced more effective cardioprotection in type-II diabetic hearts damaged with I/R through modulation of autophagy flux and improving mitochondrial function in risk areas of $\mathrm{I} / \mathrm{R}$ hearts. Even though, further investigations are required to clarify the role of different mechanisms and signaling pathways affected by combined conditioning to protect the myocardium via modulation of autophagy and mitochondrial function, particularly in the in vivo models of $\mathrm{I} / \mathrm{R}$ injury.

\section{Declarations}

\section{Ethical approval}

All experimental protocols and procedures were approved by the Institutional Animal Ethical Committee at the Faculty of Medicine of Tabriz University of Medical Sciences (Ethical code: IR.NIMAD.REC.1396.029).

\section{Consent to Participate}

Not applicable.

\section{Consent to Publish}

Not applicable 


\section{Authors Contributions}

MA and BM performed the experimental tests, gathered and analyzed the data, and drafted the manuscript. AA and NA contributed in interpretation of the results. AJ contributed in manuscript drafting and interpretation of the results. RB did the study design, supervised the whole project, contributed in interpretation of the results and finalized the manuscript editing and critically revised the manuscript. All gave final approval and agree to be accountable for all aspects of work ensuring integrity and accuracy. The authors declare that all data were generated in-house and that no paper mill was used.

\section{Funding}

The author(s) disclosed receipt of the following financial support for the research, authorship, and/or publication of this article: This work has been supported by a grant from National Institute for Medical Research Development (NIMAD).

\section{Competing Interests}

The author(s) declared no potential conflicts of interest with respect to the research, authorship, and/or publication of this article.

\section{References}

1. Zhao D, Yang J, Yang L, Insights for oxidative stress and mTOR signaling in myocardial ischemia/reperfusion injury under diabetes. Oxidative Medicine and Cellular Longevity (2017) 2017

2. Mokhtari B et al (2020) Human amniotic membrane mesenchymal stem cells-conditioned medium attenuates myocardial ischemia-reperfusion injury in rats by targeting oxidative stress. Iranian Journal of Basic Medical Sciences 23(11):1453

3. Bayrami G et al (2018) Effect of ischemic postconditioning on myocardial function and infarct size following reperfusion injury in diabetic rats pretreated with vildagliptin. J Cardiovasc Pharmacol Therap 23(2):174-183

4. Badalzadeh R et al (2017) Chronic type-I diabetes could not impede the anti-inflammatory and antiapoptotic effects of combined postconditioning with ischemia and cyclosporine $A$ in myocardial reperfusion injury. Journal of physiology biochemistry 73(1):111-120

5. Takagi H, Matsui Y, Sadoshima J (2007) The role of autophagy in mediating cell survival and death during ischemia and reperfusion in the heart. Antioxid Redox Signal 9(9):1373-1382

6. Lejay A et al (2016) Ischemia reperfusion injury, ischemic conditioning and diabetes mellitus. J Mol Cell Cardiol 91:11-22

7. Ouyang C, You J, Xie Z (2014) The interplay between autophagy and apoptosis in the diabetic heart. J Mol Cell Cardiol 71:71-80

8. Matsui $Y$ et al (2007) Distinct roles of autophagy in the heart during ischemia and reperfusion: roles of AMP-activated protein kinase and Beclin 1 in mediating autophagy. Circulation research 
100(6):914-922

9. Schiattarella GG, Hill JA (2016) Therapeutic targeting of autophagy in cardiovascular disease. J Mol Cell Cardiol 95:86-93

10. Yan W et al (2013) Impaired mitochondrial biogenesis due to dysfunctional adiponectin-AMPK-PGC1a signaling contributing to increased vulnerability in diabetic heart. Basic Res Cardiol 108(3):329

11. Yu L et al (2017) Melatonin ameliorates myocardial ischemia/reperfusion injury in type 1 diabetic rats by preserving mitochondrial function: role of AMPK-PGC-1a-SIRT3 signaling. Sci Rep 7(1):1-13

12. Miki T et al (2012) Effects of diabetes on myocardial infarct size and cardioprotection by preconditioning and postconditioning. Cardiovascular diabetology 11(1):1-13

13. Ghibu S et al (2009) Antioxidant properties of an endogenous thiol: alpha-lipoic acid, useful in the prevention of cardiovascular diseases. J Cardiovasc Pharmacol 54(5):391-398

14. Li C-j et al (2009) Attenuation of myocardial apoptosis by alpha-lipoic acid through suppression of mitochondrial oxidative stress to reduce diabetic cardiomyopathy. Chin Med J 122(21):2580-2586

15. Ghelani H, Razmovski-Naumovski V, Nammi S, Chronic treatment of (R)-a-lipoic acid reduces blood glucose and lipid levels in high-fat diet and low-dose streptozotocin-induced metabolic syndrome and type 2 diabetes in Sprague-Dawley rats. Pharmacology research \& perspectives, 2017. 5(3)

16. Srinivasan K et al (2005) Combination of high-fat diet-fed and low-dose streptozotocin-treated rat: a model for type 2 diabetes and pharmacological screening. Pharmacological research 52(4):313-320

17. Geloneze B et al (2009) HOMA1-IR and HOMA2-IR indexes in identifying insulin resistance and metabolic syndrome: Brazilian Metabolic Syndrome Study (BRAMS). Arquivos Brasileiros de Endocrinologia Metabologia 53(2):281-287

18. Hosseini L, Vafaee MS, Badalzadeh R (2020) Melatonin and nicotinamide mononucleotide attenuate myocardial ischemia/reperfusion injury via modulation of mitochondrial function and hemodynamic parameters in aged rats. J Cardiovasc Pharmacol Therap 25(3):240-250

19. Bayrami $G$ et al (2018) Combination of vildagliptin and ischemic postconditioning in diabetic hearts as a working strategy to reduce myocardial reperfusion injury by restoring mitochondrial function and autophagic activity. Advanced pharmaceutical bulletin 8(2):319

20. Alegria JR et al (2007) Infarct size, ejection fraction, and mortality in diabetic patients with acute myocardial infarction treated with thrombolytic therapy. American heart journal 154(4):743-750

21. Ferdinandy P, Schulz R, Baxter GF (2007) Interaction of cardiovascular risk factors with myocardial ischemia/reperfusion injury, preconditioning, and postconditioning. Pharmacological reviews 59(4):418-458

22. Ferdinandy P et al (2014) Interaction of risk factors, comorbidities, and comedications with ischemia/reperfusion injury and cardioprotection by preconditioning, postconditioning, and remote conditioning. Pharmacological reviews 66(4):1142-1174

23. Hsu H-C et al (2016) High-fat diet induces cardiomyocyte apoptosis via the inhibition of autophagy. Eur J Nutr 55(7):2245-2254 
24. Hayat SA et al (2004) Diabetic cardiomyopathy: mechanisms, diagnosis and treatment. Clin Sci 107(6):539-557

25. Xuan F et al (2017) 17-Methoxyl-7-Hydroxy-Benzene-Furanchalcone Ameliorates Myocardial Ischemia/Reperfusion Injury in Rat by Inhibiting Apoptosis and Autophagy Via the PI3K-Akt Signal Pathway. Cardiovasc Toxicol 17(1):79-87

26. Przyklenk K et al (2012) Autophagy as a therapeutic target for ischaemia/reperfusion injury? Concepts, controversies, and challenges. Cardiovascular research 94(2):197-205

27. Sciarretta $S$ et al., Is autophagy in response to ischemia and reperfusion protective or detrimental for the heart? Pediatric cardiology, 2011. 32(3): p. 275-281

28. Hao M et al (2017) Myocardial ischemic postconditioning promotes autophagy against ischemia reperfusion injury via the activation of the nNOS/AMPK/mTOR pathway. Int J Mol Sci 18(3):614

29. Wei C et al (2013) Activation of autophagy in ischemic postconditioning contributes to cardioprotective effects against ischemia/reperfusion injury in rat hearts. J Cardiovasc Pharmacol 61(5):416-422

30. Huang $C$ et al (2010) Autophagy induced by ischemic preconditioning is essential for cardioprotection. J Cardiovasc TransI Res 3(4):365-373

31. Cheng B-C et al (2013) Hypothermia may attenuate ischemia/reperfusion-induced cardiomyocyte death by reducing autophagy. Int J Cardiol 168(3):2064-2069

32. Xu J et al., Mitochondrial JNK activation triggers autophagy and apoptosis and aggravates myocardial injury following ischemia/reperfusion. Biochimica et Biophysica Acta (BBA)-Molecular Basis of Disease, 2015. 1852(2): p. 262-270

33. Zeng M et al (2013) NF-kB-mediated induction of autophagy in cardiac ischemia/reperfusion injury. Biochem Biophys Res Commun 436(2):180-185

34. Sciarretta $S$ et al (2015) Boosting autophagy in the diabetic heart: a translational perspective. Cardiovascular diagnosis therapy 5(5):394

35. Gottlieb RA, Mentzer RM Jr (2010) Autophagy during cardiac stress: joys and frustrations of autophagy. Annu Rev Physiol 72:45-59

36. Kobayashi S, Liang Q, Autophagy and mitophagy in diabetic cardiomyopathy. Biochimica et Biophysica Acta (BBA)-Molecular Basis of Disease, 2015. 1852(2): p. 252-261

37. Gonzalez CD et al (2011) The emerging role of autophagy in the pathophysiology of diabetes mellitus. Autophagy 7(1):2-11

\section{Figures}


A

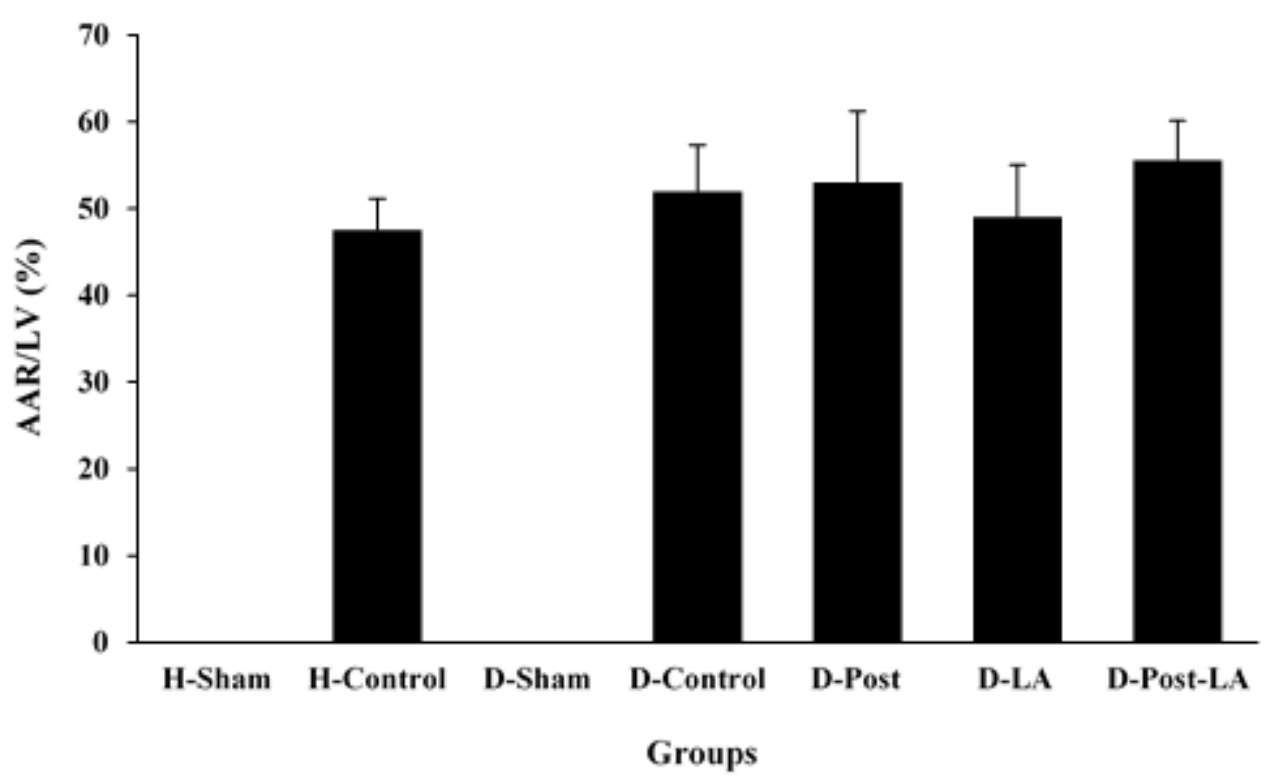

B

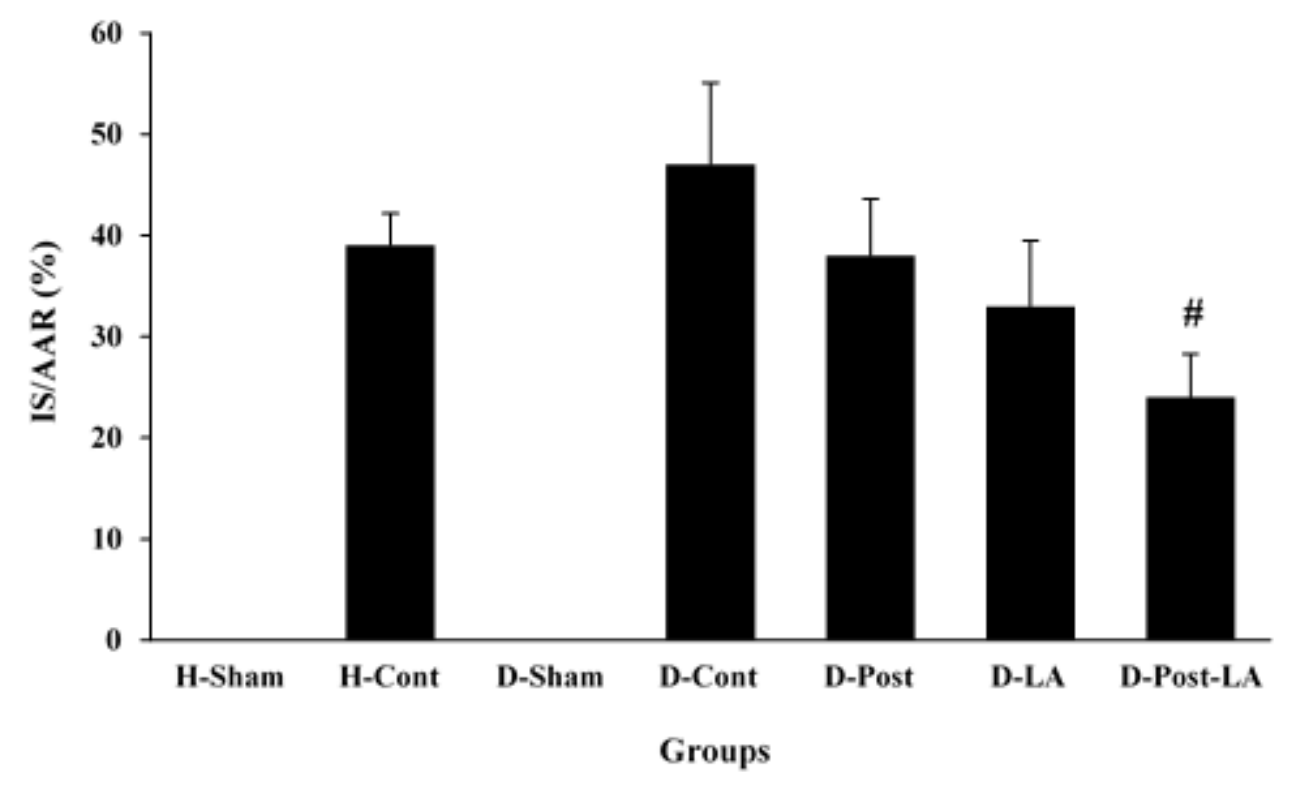

Figure 1

Area at risk (AAR) (A), and infarct size (IS) (B) percentages in experimental groups ( $\mathrm{n}=6$ for each group). The data were expressed as mean \pm SEM. (\# P < 0.05 vs. D-Cont group). H: healthy; Cont: control; D: diabetic; Post: ischemic postconditioning; LA: a-lipoic acid. 
A

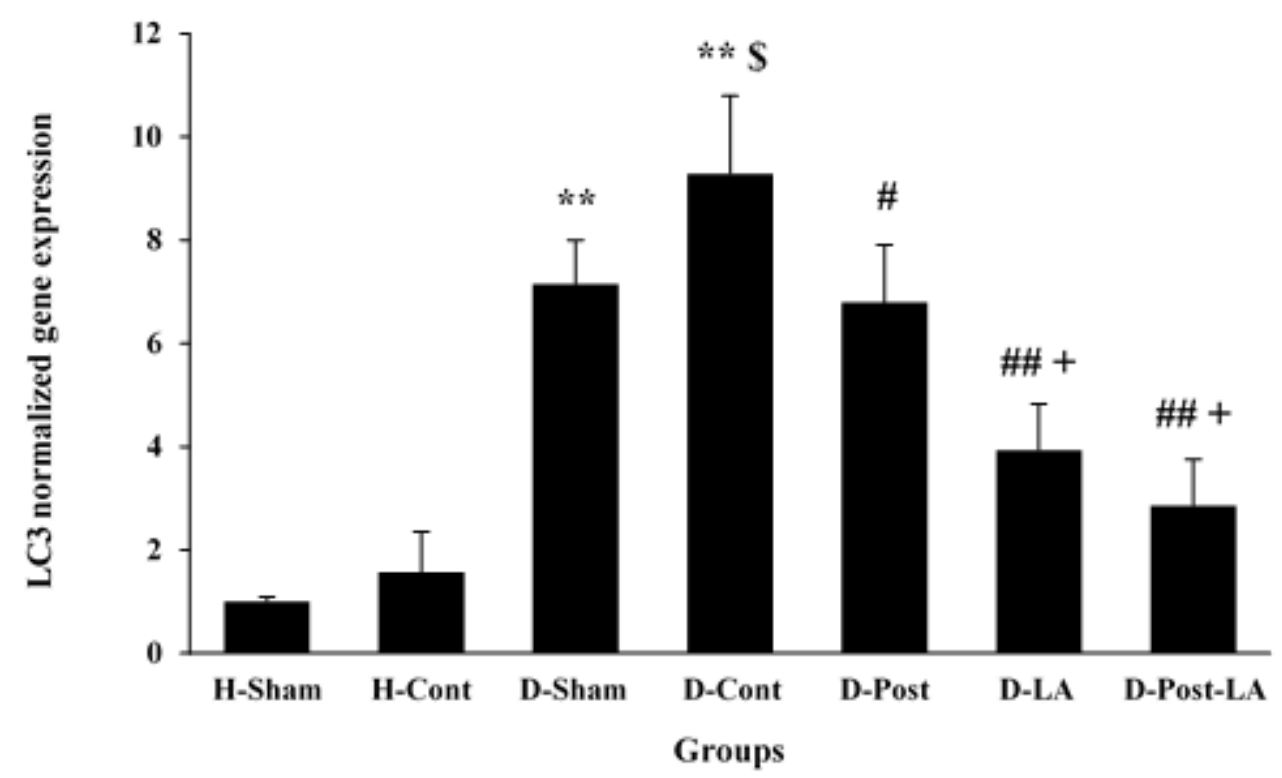

B

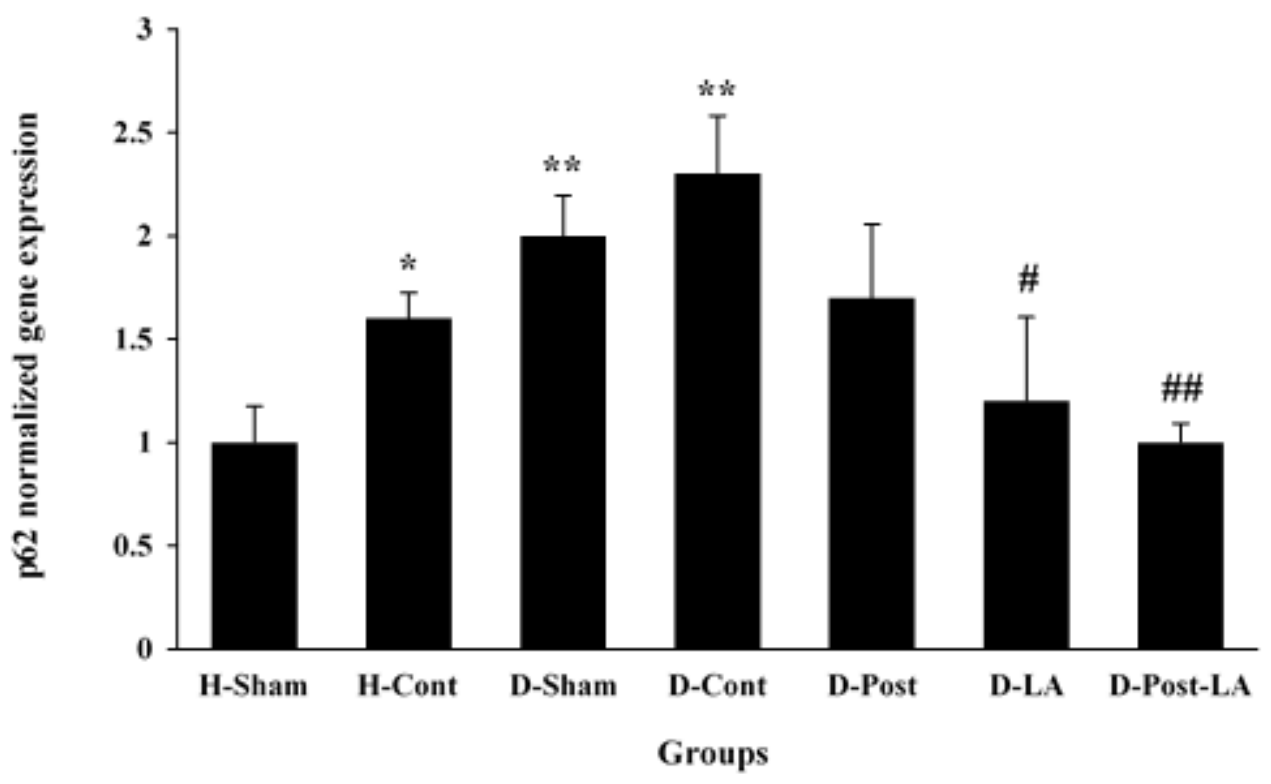

Figure 2

The expression of LC3 (A), and p62 (B) genes in experimental groups ( $n=6$ for each group). The data were expressed as mean \pm SEM. (* $P<0.05$ and ** $P<0.01$ vs. H-Sham group, $\$ P<0.05$ vs. D-Sham group, \# P < 0.05 and \#\# P < 0.01 vs. D-Cont group, + P < 0.05 vs. D-Post group). H: healthy; Cont: control; D: diabetic; Post: ischemic postconditioning; LA: a-lipoic acid. 


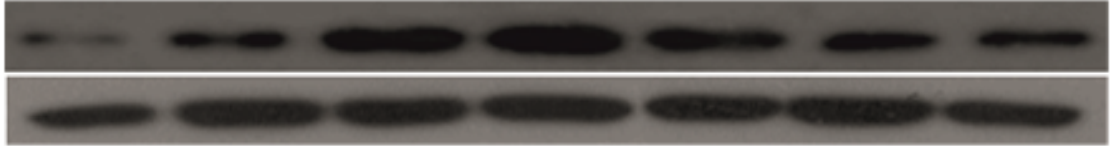

A

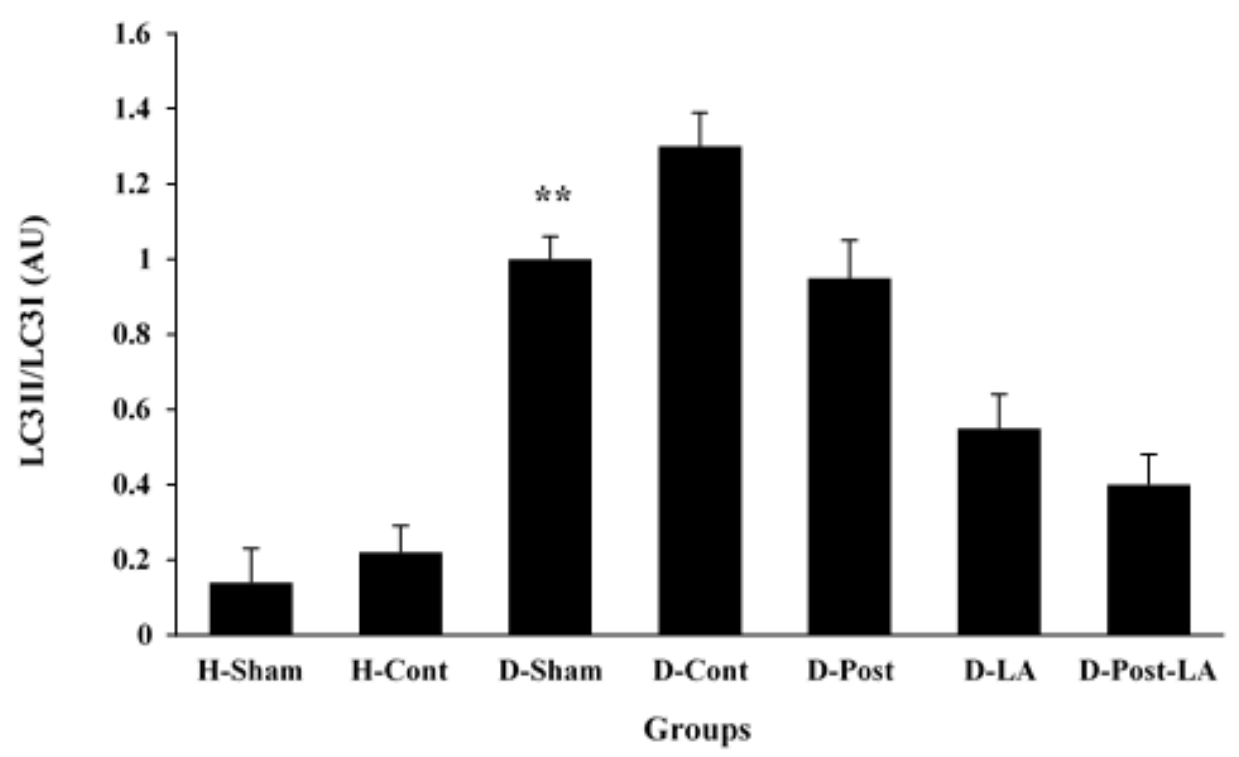

p62

$\beta$-actin

B

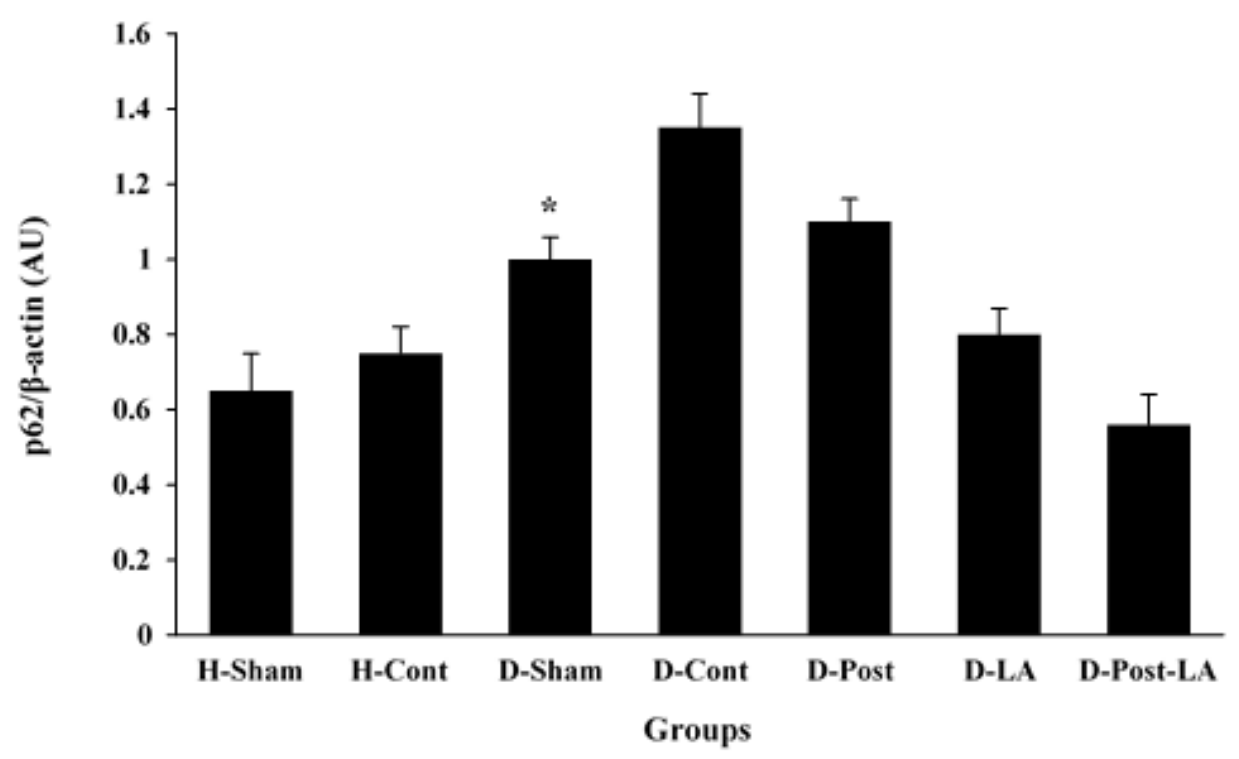

\section{Figure 3}

Changes of LC3II/LC3I ratio (A), and p62 expression (B) in experimental groups ( $\mathrm{n}=6$ for each group). The data were expressed as mean \pm SEM. ( $P<0.05$ and $* \star P<0.01$ vs. H-Sham group, $\$ P<0.05$ vs. DSham group, \# P < 0.05 and \#\# P < 0.01 vs. D-Cont group, + P < 0.05 vs. D-Post group). H: healthy; Cont: control; D: diabetic; Post: ischemic postconditioning; LA: a-lipoic acid. 
A

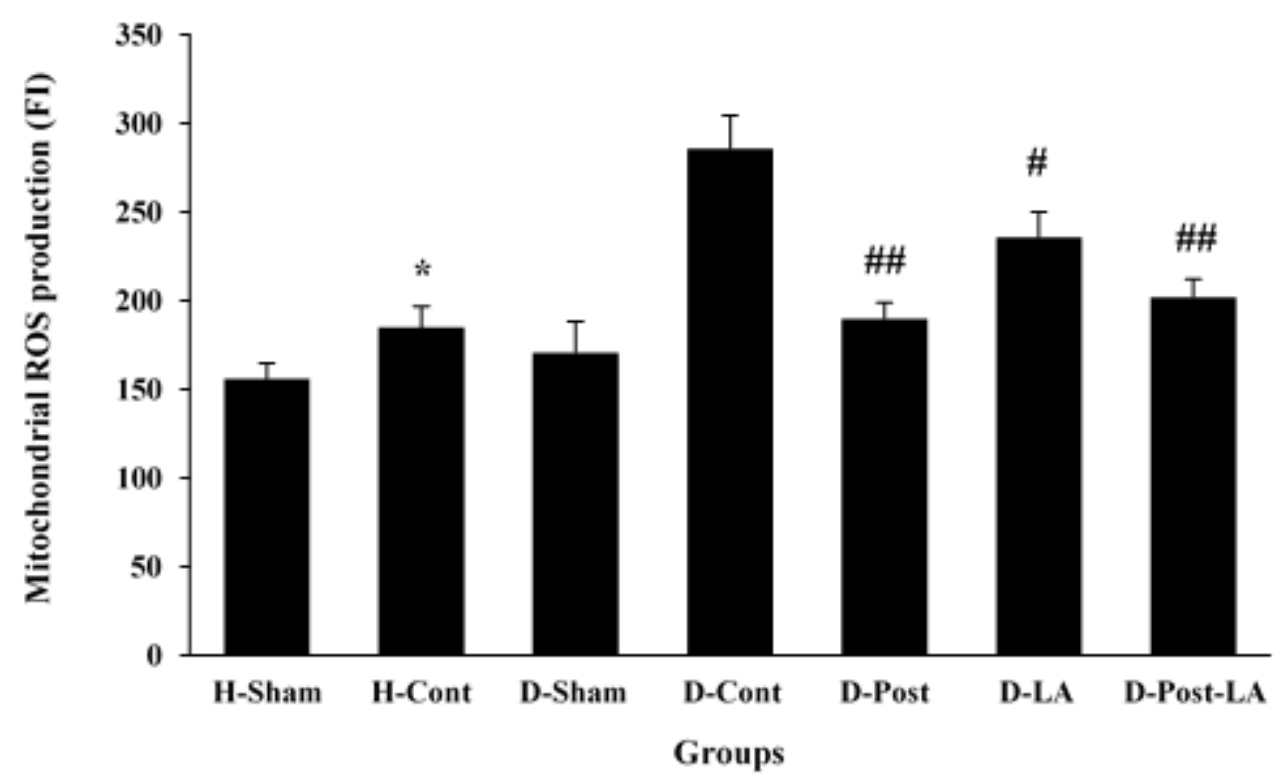

B

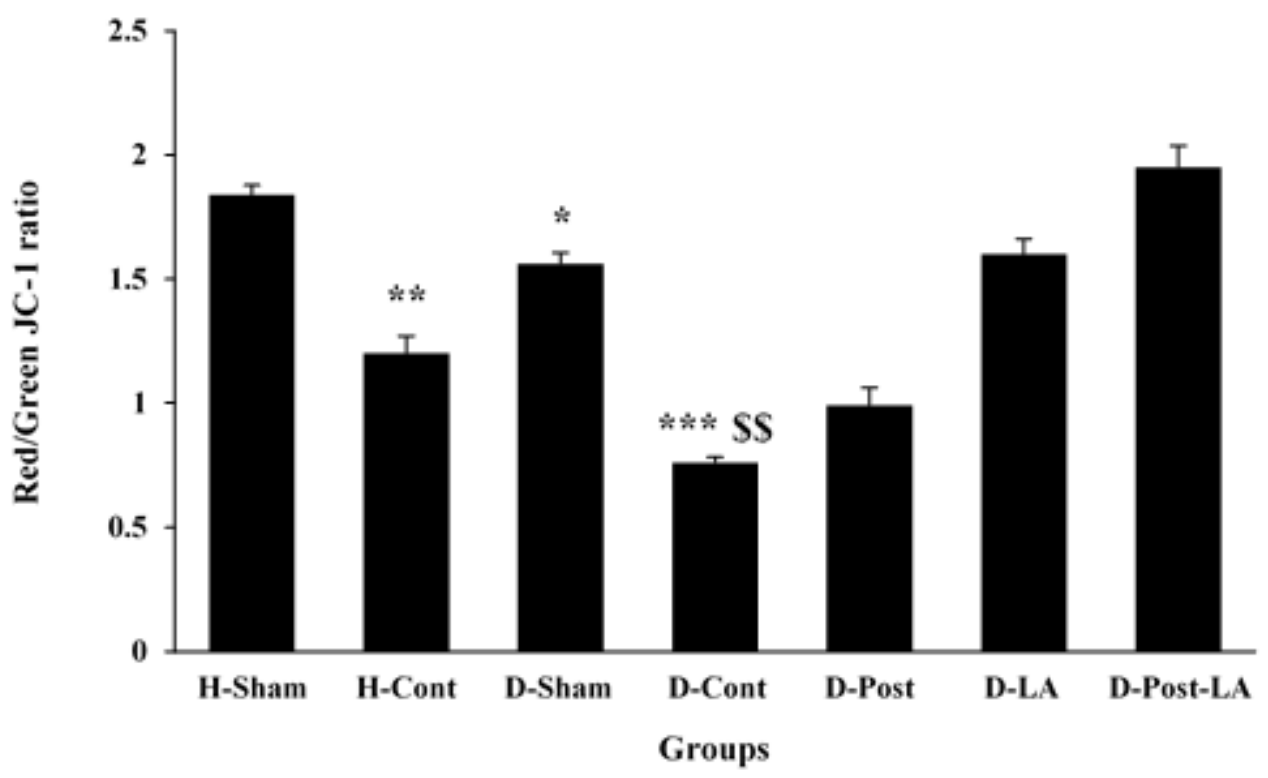

Figure 4

Mitochondrial ROS production in the heart assessed by DCFH-DA dye (A), and mitochondrial membrane potential alterations in the heart assessed by JC-1 dye (B). ( $n=6$ for each group). The data were expressed as mean \pm SEM. ( ${ }^{*}<<0.05$, ** $P<0.01$ and $* \star \star P<0.001$ vs. H-Sham group, $\$ P<0.05$ and $\$ \$$ $P<0.01$ vs. D-Sham group, \# $P<0.05$ and \#\# $P<0.01$ vs. D-Cont group, $+P<0.05$ and $++P<0.01$ vs. $D-$ Post group). H: healthy; Cont: control; D: diabetic; Post: ischemic postconditioning; LA: a-lipoic acid. 